\title{
Primary cutaneous histoplasmosis in an immunocompromised patient with long-standing rheumatoid arthritis
}

\author{
Michelle Larissa Zini Lise ${ }^{1 *}$, Henrique Luiz Staub ${ }^{2}$ \\ ${ }^{1}$ PhD, Dermatologist, Preceptor at Pontifícia Universidade Católica do Rio Grande do Sul (PUC-RS), Porto Alegre, RS, Brazil \\ ${ }^{2}$ PhD, Rheumatologist, Preceptor at PUC-RS, Porto Alegre, RS, Brazil \\ Study conducted at Hospital São Lucas, \\ Pontifícia Universidade Católica do Rio Grande do Sul (PUC-RS), Porto Alegre, RS, Brazil \\ Article received: 9/18/2016 \\ Accepted for publication: 10/19/2016 \\ *Correspondence: \\ Address: Av. Soledade, 569-912, \\ Torre Alfa \\ Porto Alegre, RS - Brazil \\ Postal code: 90470-340 \\ dramizini@yahoo.com.br \\ http://dx.doi.org/10.1590/1806-9282.62.09.816
}

\section{INTRODUCTION}

Histoplasmosis is the most common endemic fungal infection in Latin America and North America, and an important cause of endemic mycoses worldwide. The fungus is often present in soil contaminated by feces of birds and bats. ${ }^{1}$

Cutaneous manifestations of histoplasmosis can be divided into primary and secondary lesions. ${ }^{2}$ Once the fungus undergoes early hematogenous spread, exclusive cutaneous presentation is rare even in immunosuppressed patients, and thus these patients usually present severe systemic disease. ${ }^{3}$

Primary cutaneous histoplasmosis is a rare form of presentation initially described in $1947,{ }^{4}$ with only 23 cases reported in the literature to date. ${ }^{5}$

We report the case of an immunocompromised patient with rheumatoid arthritis (RA) who developed, unexpectedly, primary cutaneous histoplasmosis $(\mathrm{PCH})$.

\section{Case report}

The patient, a 52-year-old man, presented clinical, laboratory and radiological findings compatible with RA since childhood. As the disease progressed, regardless of a variety of therapies employed, he developed major deformities of the hands, wrists, elbows, hips, knees and ankles. Recently, using low-dose prednisone and methotrexate, he suddenly presented nodular ulcerated lesions on the face as seen in Figures 1 and 2. The diagnostic hypotheses were cutaneous lymphoma, leishmaniasis, sporotrichosis, cryptococcosis, cutaneous tuberculosis, histoplasmosis and tertiary syphilis. Histological examination showed diffuse granulomatous infiltrate with the presence of intracytoplasmic yeast structures (Figure 3). Curettage was performed for culture of material from the necrotic-suppurative areas of ulcerated lesions. Histoplasma capsulatum was the fungus detected by direct mycological examination (Grocott staining). The chest radiograph was normal. Blood cell count was unremarkable. After 6 months of therapy using itraconazole $300 \mathrm{mg}$ daily, the nodules on the skin of the face disappeared. The patient continued to be monitored after completion of treatment without any disease recurrence signals.

\section{Discussion}

Histoplasmosis is a disease caused by the dimorphic fungus Histoplasma capsulatum, which is common in tropical and temperate countries. The usual form of presentation is the respiratory one, second to inhalation of airborne conidia. $^{6}$

In our immunosuppressed RA patient, a disseminated form of histoplasmosis would be the expected presentation, but atypical PCH turned out to be the final diagnosis. Primary cutaneous histoplasmosis has been described in immunocompetent individuals, but reports of this form of presentation in immunocompromised are rare. Today, it is clear that the infection is the result of a complex interaction between pathogen and host.? 


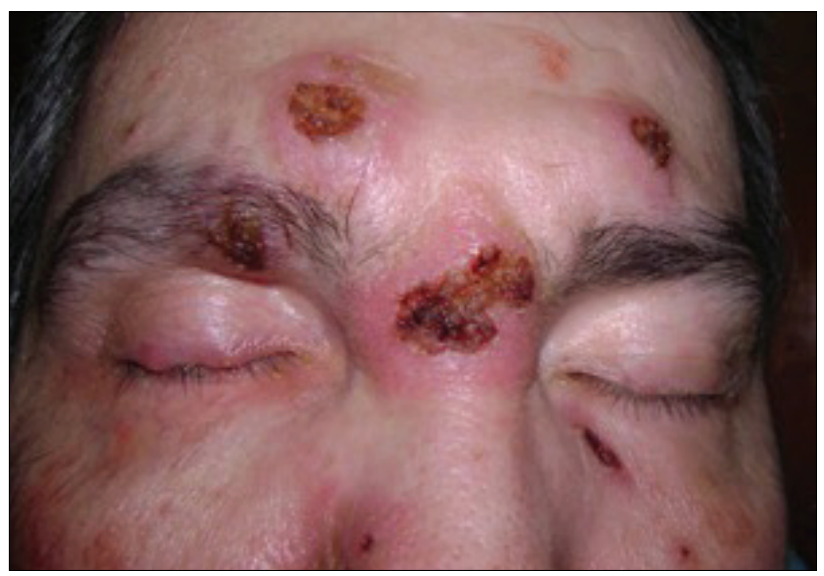

FIGURE 1 Ulcerated nodular lesions in the frontal region.

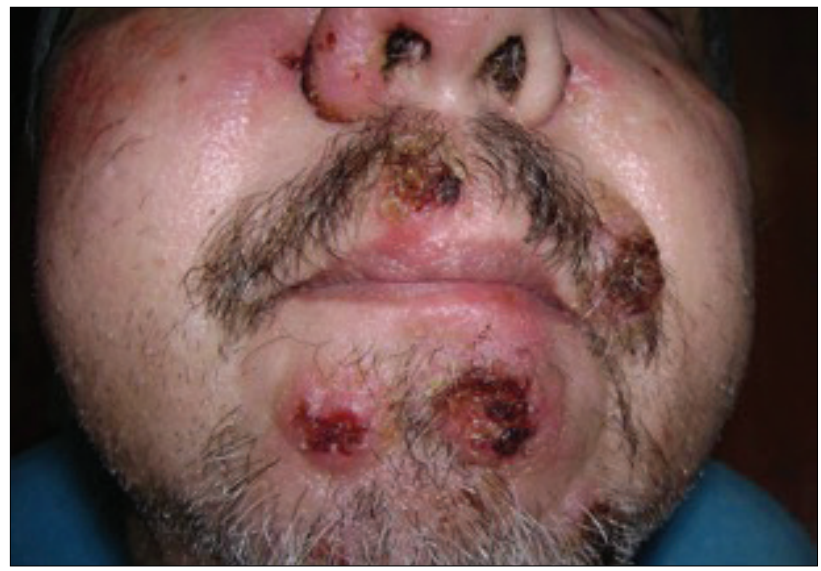

FIGURE 2 Detail of ulcerated nodular lesions in the chin region.

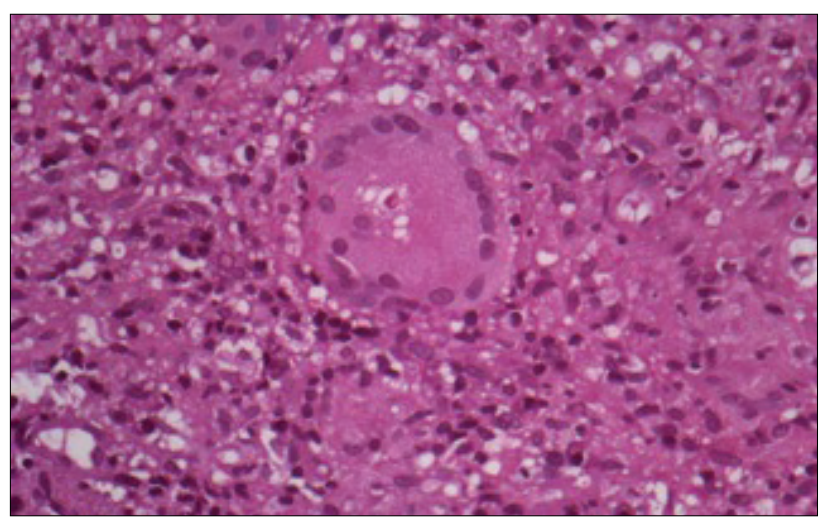

FIGURE 3 Histopathological examination, HE 400x.
The patient presented lesions with no regional lymphadenopathy and no evidence of systemic involvement. Also, Histoplasma capsulatum was isolated and identified. There were only a few descriptions of $\mathrm{PCH}$ in patients with autoimmune rheumatic diseases such as the current case. ${ }^{5}$ As previously reported, ${ }^{3}$ the use of itraconazole as therapy of choice yielded complete response.

In summary, we describe an unusual case of $\mathrm{PCH}$ in a patient with long-standing RA treated with steroids and methotrexate. We stress the need for histological and mycological examination of skin lesions suspected in such patients.

\section{ACKNOWLEDGMENTS}

We are indebted to Dr. Lúcio Bakos, Dr. Valério Aquino and Dr. André Cartell.

\section{References}

1. Cano MV, Hajjeh RA. The epidemiology of histoplasmosis: a review. Semin Respir Infect. 2001; 16(2):109-18.

2. Chanda JJ, Callen JP. Isolated nodular cutaneous histoplasmosis. The initial manifestation of recurrent disseminated disease. Arch Dermatol. 1978; 114(8):1197-80.

3. Wheat LJ, Kauffman CA. Histoplasmosis. Infect Dis Clin North Am. 2003; 17(1):1-19, vii.

4. Curtis AC, Cawley EP. Genital histoplasmosis. J Urol. 1947; 57(4):781-7.

5. Lise ML, Godinho RN, Brollo FM, Staub HL. Cutaneous ulcer in an immunosuppressed patient with adult onset Still's disease: primary cutaneous histoplasmosis? An Bras Dermatol. 2014; 89(3):532-4.

6. Saheki MN, Schubach AO, Salgueiro MM, Conceição-Silva F, Wanke B, Lazera M. [Primary cutaneous histoplasmosis: case report on an immunocompetent patient and review of the literature]. Rev Soc Bras Med Trop. 2008; 41(6):680-2

7. Sardinha JF, Tarlé RG, Fava VM, Francio AS, Ramos GB, Ferreira LC, et al. Genetic risk factors for human susceptibility to infections of relevance in dermatology. An Bras Dermatol. 2011; 86(4):708-15. 